\title{
Nonclassical Effects of Light in Fifth Harmonic Generation up to First-Order Hamiltonian Interaction
}

\author{
Rajendra Pratap, ${ }^{1}$ D. K. Giri, ${ }^{2}$ and Ajay Prasad ${ }^{3}$ \\ ${ }^{1}$ Department of Physics, R. S. More College, Vinoba Bhave University, Hazaribag, Govindpur 828109, India \\ ${ }^{2}$ Department of Physics, Sindri College, Vinoba Bhave University, Hazaribag, Sindri 828122, India \\ ${ }^{3}$ Department of Physics, P. K. R. M. College, Vinoba Bhave University, Hazaribag, Dhanbad 826004, India \\ Correspondence should be addressed to Ajay Prasad; dr.ajaypd@gmail.com
}

Received 3 November 2013; Accepted 30 December 2013; Published 9 March 2014

Academic Editors: O. Frazão and V. Matejec

Copyright (c) 2014 Rajendra Pratap et al. This is an open access article distributed under the Creative Commons Attribution License, which permits unrestricted use, distribution, and reproduction in any medium, provided the original work is properly cited.

\begin{abstract}
The nonclassical effects of light in the fifth harmonic generation are investigated by quantum mechanically up to the first-order Hamiltonian interaction. The coupled Heisenberg equations of motion involving real and imaginary parts of the quadrature operators are established. The occurrence of amplitude squeezing effects in both quadratures of the radiation field in the fundamental mode is investigated and found to be dependent on the selective phase values of the field amplitude. The photon statistics in the fundamental mode have also been investigated and found to be sub-Poissonian in nature. It is observed that there is no possibility to produce squeezed light in the harmonic mode up to first-order Hamiltonian interaction. Further, we have found that the normal squeezing in the harmonic mode directly depends upon the fifth power of the field amplitude of the initial pump field up to second-order Hamiltonian interaction. This gives a method of converting higher-order squeezing in the fundamental mode into normal squeezing in the harmonic mode and vice versa. The analytic expression of fifth-order squeezing of the fundamental mode in the fifth harmonic generation is established.
\end{abstract}

\section{Introduction}

The nonclassical effects like squeezing and sub-Poissonian photon statistics of light [1-3], which is a purely quantum mechanical phenomenon [4-6], have attracted considerable attention owing to its low-noise property [7-9] with applications in high quality telecommunication [10], quantum cryptography [11, 12], and so forth. Squeezing has been either experimentally observed or theoretically predicted in a variety of nonlinear optical processes, such as harmonic generation $[13,14]$, multiwave mixing processes [15-18], Raman [19-21], and hyper-Raman [22]. Hong and Mandel [23, 24], Hillery [25-27], and Zhan [28] have introduced the notion of amplitude squeezing of the quantized electromagnetic field in various nonlinear optical processes. Squeezing and photon statistical effect of the field amplitude in optical parametric and in Raman and hyper-Raman scattering processes have also been reported by Peěrina et al. [29]. Higher-order sub-Poissonian photon statistical of light have also been studied by Kim and Yoon [30]. Recently, Prakash and Mishra
$[31,32]$ have reported the higher-order sub-Poissonian photon statistics and their use in detection of higher-order squeezing. Furthermore, higher-order amplitude squeezing with dependence on photon number in fourth and fifth harmonic generation has also been investigated by Gill et al. [33]. The nonclassical phenomena squeezing of radiation and photon statistics effects are expected to manifest itself in optical processes in which the nonlinear response of the system to the radiation field plays a great role. It also represents a new type of quantum state of the electromagnetic field and it has always been of interest to the research community in the fields of quantum optics, nonlinear optics, atomic physics, molecular physics, and biological physics; hence their study can be expected to lead to new fundamental insights.

The objective of this paper is to study nonclassical effects of the light in the fundamental mode including harmonic mode in fifth harmonic generation process under shorttime approximation based on a fully quantum mechanical approach up to first order Hamiltonian interaction in $g t$. The 
paper is organized as follows. Section 2 gives the definition of squeezing and sub-Poissonian states of light. We establish the analytic expression of selective phase angle dependent amplitude squeezing and sub-Poissonian light in the fundamental mode up to first order in $g t$ in Section 3. The photon statistics of the pump mode in this process have also been incorporated in this section and found to be sub-Poissonian in nature. In Section 4, we study the occurrence of amplitude squeezing effects in both quadratures of the radiation field in the harmonic mode and they were found to be dependent on fifth-order squeezing of the fundamental mode. Finally, we conclude the paper in Section 5.

\section{Definition of Nonclassical States of Light}

Squeezed states of light are characterized by reduced quantum fluctuations in one quadrature of the field at the expense of the increased fluctuations in the other quadrature. It is possible to characterize the amplitude by its real and imaginary parts as

$$
X_{1}=\frac{1}{2}\left(A+A^{\dagger}\right), \quad X_{2}=\frac{1}{2 i}\left(A-A^{\dagger}\right),
$$

where $A \equiv A(t)$ and $A^{\dagger} \equiv A^{\dagger}(t)$ are the slowly varying operators because the interaction between modes, it induces a slower dependence on time as compared to fast variation of $a(t) \propto \exp (-i \omega t)$ and $a^{\dagger}(t) \propto \exp (i \omega t)$ that are useful in discussing squeezing effects $[34,35]$. For a single mode of the electromagnetic field with frequency $\omega$ and creation (annihilation) operators $a^{\dagger}(a)$, the slowly varying operators defined by

$$
A(t)=a(t) \exp (i \omega t), \quad A^{\dagger}(t)=a^{\dagger}(t) \exp (-i \omega t) .
$$

The operators defined by (1) do not commute and obey the commutation relation

$$
\left[X_{1}, X_{2}\right]=\frac{i}{2}
$$

and, as a result, they satisfy the uncertainty relation $(\hbar=1)$

$$
\Delta X_{1} \Delta X_{2} \geq \frac{1}{4}
$$

where $\Delta X_{1}$ and $\Delta X_{2}$ are the uncertainties in the quadrature operators $X_{1}$ and $X_{2}$, respectively. A quantum state is squeezed in the $X_{1}$ direction if $\Delta X_{1}<1 / 2$ and is squeezed in the $X_{2}$ direction if $\Delta X_{2}<1 / 2$.

In order to define higher-order squeezing i.e. amplitude squared squeezing $[25,26]$, we represent the real and imaginary parts of the square of the field amplitude in terms of operators $Y_{1}$ and $Y_{2}$ as

$$
Y_{1}=\frac{1}{2}\left(A^{2}+A^{\dagger 2}\right), \quad Y_{2}=\frac{1}{2 i}\left(A^{2}-A^{\dagger 2}\right) \text {. }
$$

The operators defined by (5) do not commute and obey the commutation relation

$$
\left[Y_{1}, Y_{2}\right]=i\left(2 N_{A}+1\right) \text {. }
$$

The commutation relation of (6) leads to the uncertainty relation

$$
\Delta Y_{1} \Delta Y_{2} \geq\left\langle\left(N_{A}+\frac{1}{2}\right)\right\rangle
$$

where $\Delta Y_{1}$ and $\Delta Y_{2}$ are the uncertainties in the quadrature operators $Y_{1}$ and $Y_{2}$, respectively. A quantum state is squeezed in the $Y_{1}$ direction if $\left(\Delta Y_{1}\right)^{2}<\left\langle\left(N_{A}+1 / 2\right)\right\rangle$ and is squeezed in the $Y_{2}$ direction if $\left(\Delta Y_{2}\right)^{2}<\left\langle\left(N_{A}+1 / 2\right)\right\rangle$.

Similarly, it is possible to characterize the fifth power of the field amplitude [22] by its real and imaginary parts as

$$
G_{1}=\frac{1}{2}\left(A^{5}+A^{\dagger 5}\right), \quad G_{2}=\frac{1}{2 i}\left(A^{5}-A^{\dagger 5}\right) .
$$

The operators defined by (8) do not commute and obey the commutation relation

$$
\begin{aligned}
{\left[G_{1}, G_{2}\right] } & =\frac{i}{2}\left(25 N_{A}^{4}+50 N_{A}^{3}+275 N_{A}^{2}+250 N_{A}+120\right) \\
& =\frac{i}{2}\left(G_{\{12\}}\right),
\end{aligned}
$$

where we introduce the auxiliary function $\left(G_{\{12\}}\right)$ instead of $\left(25 N_{A}^{4}+50 N_{A}^{3}+275 N_{A}^{2}+250 N_{A}+120\right)$.

And the commutation relation of (9) follows the uncertainty relation as

$$
\Delta G_{1} \Delta G_{2} \geq \frac{1}{4}\left\langle\left(G_{\{12\}}\right)\right\rangle .
$$

Hence the fifth-order squeezing is said to exist if

$$
\left(\Delta G_{1}\right)^{2} \quad \text { or } \quad\left(\Delta G_{2}\right)^{2}<\frac{1}{4}\left\langle\left(G_{\{12\}}\right)\right\rangle
$$

The quantum effect of sub-Poissonian photon statistics is the reduction of quantum fluctuations in photon number which is reflected by an increase of fluctuations of phase of the field. Hence the photon number uncertainty [36] is

$$
\left\langle\left(\Delta N_{A}\right)^{2}\right\rangle<\left\langle N_{A}\right\rangle \text {. }
$$

\section{Nonclassical Effects of Light in the Fundamental Mode}

Fifth harmonic generation is a process in which an incident laser beam of the fundamental frequency $\omega_{1}$ interacts with a nonlinear medium to produce the harmonic frequency at $\omega_{2}=5 \omega_{1}$.

The present model, shown in Figure 1, has been adopted from the works of Chang et al. [37] who have studied the optimal combination of nonlinear optical crystals for the fifth harmonic generation and $\mathrm{Ni}$ et al. [38] have reported the comparative experimental investigation on third and fifth harmonic generation by mid-infrared ultrafast laser pulses. This model is chosen to make a realistic one and our discussions hold for all similar models. 


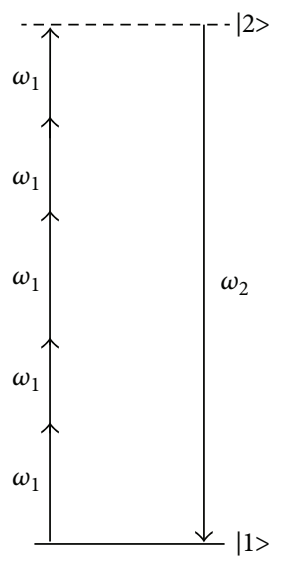

FIGURE 1: Fifth harmonic generation energy level model.

In this model (Figure 1), the interaction is looked upon as a process involving absorption of five pump photons of frequency $\omega_{1}$ each and the system going from state $|1\rangle$ to state $|2\rangle$ and emission of one photon of frequency $\omega_{2}$ and the atomic system is finally coming back to the initial state $|1\rangle$.

The Hamiltonian for this process can be written as $(\hbar=1)$

$$
H=\omega_{1} a^{\dagger} a+\omega_{2} b^{\dagger} b+g\left(a^{5} b^{\dagger}+a^{\dagger 5} b\right)
$$

where $a^{\dagger}(a)$ and $b^{\dagger}(b)$ are the creation (annihilation) operators of the pump field ( $A$-mode) and harmonic field ( $B$ mode), respectively, and $g$ is the coupling constant in the interaction Hamiltonian, which is assumed to be real, describes the coupling between the two modes of the order of $10^{2}-10^{4}$ per second and is proportional to the nonlinear susceptibility of the medium as well as the complex amplitude of the pump field $[39,40]$. However, to take care of complex $g$, we have used $|g|^{2}$ in the place of $g^{2}$ as we are not considering the phase terms. In the case of phase matching, $g$ can also be treated as real [4]. Furthermore, in an earlier publication [41], it is shown that the real part of $g$ plays an important role in the generalization of squeezed light.

Since the time dependence of free modes $(g=0)$ is simply proportional to $\exp \left(i \omega_{1} t\right)$ or $\exp \left(i \omega_{2} t\right)$. The interaction between modes $(g \neq 0)$ induces a slower dependence on time and we can write $A(t)=a(t) \exp \left(i \omega_{1} t\right)$ and $B(t)=$ $b(t) \exp \left(i \omega_{2} t\right)$ with the relation $\omega_{2}=5 \omega_{1}$, where the operators $A(t)$ and $B(t)$ vary slowly in time. Later it will be evident that $A(t)$ and $B(t)$ indeed depend on a scaled time " $g t$ " (not on $t$ ), thus satisfying their slow variation of the operators $A(t)$ and $B(t)$ as compared to fast variation of $a(t)$ and $b(t)$ since $g \ll \omega_{1}, \omega_{2}$ usually.

Using the Hamiltonian interaction of (13) in the coupled Heisenberg equation of motion, we have

$$
\dot{A}(t)=\frac{\partial A(t)}{\partial t}+i[H, A(t)] \quad(\hbar=1)
$$

where the dot denotes time derivative and the partial time derivative term and Hamiltonian interaction in Heisenberg picture is represented as

$$
\begin{gathered}
\frac{\partial A(t)}{\partial t}=i \omega_{1} a(t) \exp \left(i \omega_{1} t\right)=i \omega_{1} A(t), \\
i[H, A(t)]=-i \omega_{1} A(t)-5 i g t A^{\dagger 4}(t) B(t) .
\end{gathered}
$$

Using the values in (14), we obtain Heisenberg's equations of motion for the slowly varying amplitude operator $A(t)$ up to the first-time derivative $[25,28]$ as

$$
\dot{A}(t)=-5 i g A^{\dagger 4}(t) B(t) .
$$

Similarly, we have

$$
\dot{B}(t)=-i g A^{5}(t) .
$$

Since the general time-dependent solution of the set of coupled differential equations (16) and (17) is not available, let us approximately solve them in the short-time limit. Note that the system evolution during a short period of time is practically relevant because the actual interaction is in fact very short. Hence the interaction time is taken to be short and to be of the order of $10^{-10} \mathrm{sec}$ and a nanosecond or picosecond pulse laser can be used as the pump field. For real physical situation in the short-time scale $g t \ll 1\left(g t \sim 10^{-6}\right)$ and when the number of photons is very large $\left(|\alpha|^{2} \gg 1\right)$, it is possible to obtain much simpler approximate analytical formulas describing the variances. Expanding $A(t)$ around the initial time $t=0$ in Taylor's expansion [28] and confining ourselves to terms up to first order in " $t$," we have

$$
A(t)=A(0)+t \dot{A}(0)+\cdots .
$$

In (18) the operators $A(0) \equiv A$ and $\left.\dot{A}(0) \equiv(\partial A(t) / \partial t)\right|_{t=0}$ without an argument are opeators evaluated at $t=0$. This convention is followed throughout the paper.

Using (16) in (18), we obtain up to first order in " $g t$ "

$$
A(t)=A(0)-5 i g t A^{\dagger 4}(0) B(0)=A-5 i g t A^{\dagger 4} B .
$$

Equation (19) indicates that $A(t)$ depends on $g t$ rather than on $t$ revealing the much slower variation of $A(t)$ as compared to the fast variation of $a(t)$.

Then in reversal order,

$$
A^{\dagger}(t)=A^{\dagger}+5 i g t A^{4} B^{\dagger} .
$$

Similarly,

$$
\begin{gathered}
B(t)=B-i g t A^{5}, \\
B^{\dagger}(t)=B^{\dagger}+i g t A^{\dagger 5} .
\end{gathered}
$$

In order to examine the amplitude squeezing of the fundamental mode $A$, as a function of time, we define quadrature components by

$$
\begin{aligned}
& X_{1 A}(t)=\frac{1}{2}\left[A(t)+A^{\dagger}(t)\right], \\
& X_{2 A}(t)=\frac{1}{2 i}\left[A(t)-A^{\dagger}(t)\right] .
\end{aligned}
$$


Using (19) and (20) in (22), we obtain

$$
\begin{aligned}
& X_{1 A}(t)=\frac{1}{2}\left[\left(A+A^{\dagger}\right)-5 i g t\left(A^{\dagger 4} B-A^{4} B^{\dagger}\right)\right], \\
& X_{2 A}(t)=\frac{1}{2 i}\left[\left(A-A^{\dagger}\right)-5 i g t\left(A^{\dagger 4} B+A^{4} B^{\dagger}\right)\right] .
\end{aligned}
$$

Now, we assume an initial quantum state as a product of coherent states $\mid \alpha>$ for the fundamental mode $A$ and $\mid \beta>$ for the harmonic mode $B$; that is,

$$
|\psi>=| \alpha>_{A} \mid \beta>_{B}
$$

Using (25) in (23), we obtain the expectation value as

$$
\begin{gathered}
\left\langle\psi\left|X_{1 A}^{2}(t)\right| \psi\right\rangle=\frac{1}{4}\left[\alpha^{2}+\alpha^{* 2}+2|\alpha|^{2}+1-10\right. \text { igt } \\
\times\left(|\alpha|^{2} \alpha^{* 3} \beta+2 \alpha^{* 3} \beta-2 \alpha^{3} \beta^{*}\right. \\
\left.\left.\quad-|\alpha|^{2} \alpha^{3} \beta^{*}+\alpha^{* 5} \beta-\alpha^{5} \beta^{*}\right)\right], \\
\left\langle\psi\left|X_{1 A}(t)\right| \psi\right\rangle^{2}=\frac{1}{4}\left[\alpha^{2}+\alpha^{* 2}+2|\alpha|^{2}-10 i g t\right. \\
\times\left(|\alpha|^{2} \alpha^{* 3} \beta+\alpha^{* 5} \beta-\alpha^{5} \beta^{*}\right. \\
\left.\left.-|\alpha|^{2} \alpha^{3} \beta^{*}\right)\right] .
\end{gathered}
$$

Hence, the field variance is

$$
\begin{aligned}
{\left[\Delta X_{1 A}(t)\right]^{2} } & =\left\langle X_{1 A}^{2}(t)\right\rangle-\left\langle X_{1 A}(t)\right\rangle^{2} \\
& =\frac{1}{4}\left[1-10 \operatorname{igt}\left(2 \alpha^{* 3} \beta-2 \alpha^{3} \beta^{*}\right)\right] .
\end{aligned}
$$

Equations (4) and (27) yield

$$
\left[\Delta X_{1 A}(t)\right]^{2}-\frac{1}{4}=10|g t|\left|\alpha^{3} \beta\right| \sin \left(\theta_{2}-3 \theta_{1}\right)
$$

where $\left\langle N_{A}\right\rangle=\left\langle A^{\dagger} A\right\rangle=|\alpha|^{2}$ and $\left\langle N_{B}\right\rangle=\left\langle B^{\dagger} B\right\rangle=|\beta|^{2}$ are the photon number operators in the modes $A$ and $B$, respectively, and $\alpha=|\alpha| \exp \left(i \theta_{1}\right)$ and $\beta=|\beta| \exp \left(i \theta_{2}\right)$ are dimensionless complex numbers; $\theta_{1}$ and $\theta_{2}$ are the phase angles; $\alpha^{*}, \beta^{*}$ denote the complex conjugate of $\alpha$ and $\beta$, respectively. From (28) it is found that the squeezing of $X_{1 A}$ will occur whenever $\sin \left(\theta_{2}-3 \theta_{1}\right)<0$.

Similarly, using (24) and (25) for $X_{2 A}$ direction, we have

$$
\left[\Delta X_{2 A}(t)\right]^{2}-\frac{1}{4}=-10|g t|\left|\alpha^{3} \beta\right| \sin \left(\theta_{2}-3 \theta_{1}\right) \text {. }
$$

The right-hand side of (29) is negative when $\sin \left(\theta_{2}-\right.$ $\left.3 \theta_{1}\right)>0$, showing the existence of normal squeezing in the fundamental mode up to first order in $g t$ in the fifth harmonic generation process. Equations (28) and (29) show that only one quadrature can be squeezed at a time; that is, it follows Heisenberg's uncertainty principle.

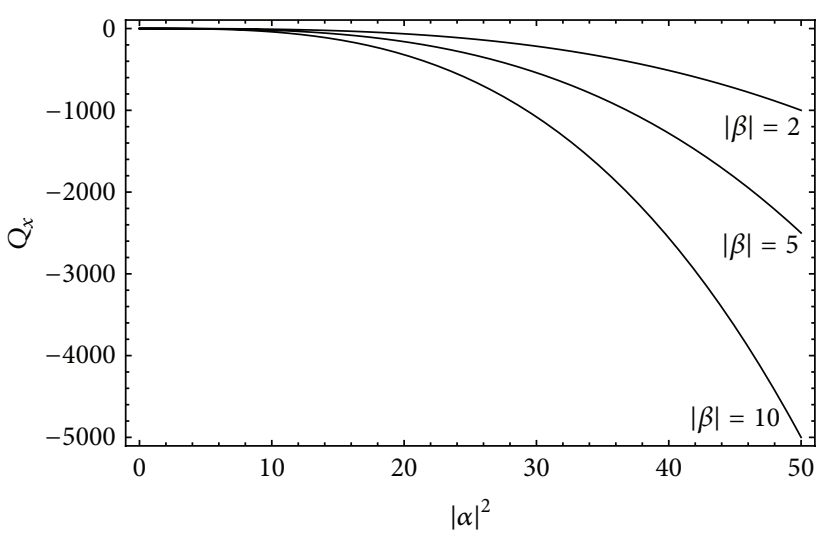

FIGURE 2: Dependence of degree of normal squeezing $Q_{x}$ with $|\alpha|^{2}$ and $|\beta|$ in fifth harmonic generation (when $|g t|=10^{-4}$ and $\theta_{1}=0$ and $\theta_{2}=\pi / 2$ ).

To measure the degree of amplitude squeezing, we define the normalized parameter [30] of (29) as

$$
Q_{x}=\frac{\left[\Delta X_{2 A}(t)\right]^{2}-1 / 4}{1 / 4}=-40|g t|\left|\alpha^{3} \beta\right| \sin \left(\theta_{2}-3 \theta_{1}\right) \text {. }
$$

Hence the degree of amplitude squeezing of the fundamental mode is found to be dependent directly on the phase angle of the field amplitude up to the first-order Hamiltonian interaction in $g t$.

We plot a graph of (30) between the normalized parameter of squeezing $Q_{x}$ and the photon number $|\alpha|^{2}$ with different values of $|\beta|$ in Figure 2.

Figure 2 shows that the normal squeezing increases nonlinearly with the increase the value of $|\alpha|^{2}$. We observe that when higher the value of $|\beta|$, then the squeezing increases and it lowers the depth of classicality of the field amplitude. It shows that the degree of normal squeezing depends directly upon the photon number of the fundamental mode as well as of the harmonic mode.

Similarly for studying one of the class of higher-order squeezing like squeezing of amplitude-squared of the field of the fundamental mode as a function of time, we define quadrature components for the pump mode as

$$
\begin{gathered}
Y_{1 A}(t)=\frac{1}{2}\left[A^{2}(t)+A^{\dagger 2}(t)\right], \\
Y_{2 A}(t)=\frac{1}{2 i}\left[A^{2}(t)-A^{\dagger 2}(t)\right] .
\end{gathered}
$$

Using (19) and (20) in (31), we get

$$
\begin{aligned}
Y_{1 A}(t)=\frac{1}{2}[ & A^{2}+A^{\dagger 2}-10 i g t \\
& \left.\times\left(A^{\dagger 4} A B+2 A^{\dagger 3} B-A^{\dagger} A^{4} B^{\dagger}-2 A^{3} B^{\dagger}\right)\right] .
\end{aligned}
$$


Using (25) in (33), we obtain the expectation value as

$$
\begin{aligned}
& \left\langle\psi\left|Y_{1 A}^{2}(t)\right| \psi\right\rangle \\
& =\frac{1}{4}\left[\alpha^{4}+\alpha^{* 4}+2|\alpha|^{4}+4|\alpha|^{2}+2\right. \\
& -20 i g t\left(|\alpha|^{6} \alpha^{*} \beta-|\alpha|^{6} \alpha \beta^{*}+6|\alpha|^{4} \alpha^{*} \beta\right. \\
& -6|\alpha|^{4} \alpha \beta^{*}+12|\alpha|^{2} \alpha^{*} \beta-12|\alpha|^{2} \alpha \beta^{*} \\
& +|\alpha|^{2} \alpha^{* 5} \beta-|\alpha|^{2} \alpha^{5} \beta^{*}+3 \alpha^{* 5} \beta-3 \alpha^{5} \beta^{*} \\
& \left.\left.+6 \alpha^{*} \beta-6 \alpha \alpha^{*}\right)\right] \\
& \left\langle\psi\left|Y_{1 A}(t)\right| \psi\right\rangle^{2} \\
& =\frac{1}{4}\left[\alpha^{4}+\alpha^{* 4}+2|\alpha|^{4}-20 i g t\right. \\
& \times\left(2|\alpha|^{4} \alpha^{*} \beta-2|\alpha|^{4} \alpha \beta^{*}+|\alpha|^{6} \alpha^{*} \beta-|\alpha|^{6} \alpha \beta^{*}\right. \\
& \left.\left.+|\alpha|^{2} \alpha^{* 5} \beta-|\alpha|^{2} \alpha^{5} \beta^{*}+2 \alpha^{* 5} \beta-2 \alpha^{5} \beta^{*}\right)\right] .
\end{aligned}
$$

And the expectation value of the time-dependent mean photon number is

$$
\left\langle N_{A}(t)\right\rangle=|\alpha|^{2}-5 i g t\left(\alpha^{* 5} \beta-\alpha^{5} \beta^{*}\right) .
$$

Using (34)-(35) in (7), we get

$$
\begin{aligned}
{\left[\Delta Y_{1 A}(t)\right]^{2}-\left\langle N_{A}(t)+1 / 2\right\rangle=} & 40|g t||\alpha \beta| \sin \left(-\theta_{1}+\theta_{2}\right) \\
& \times\left\{\left(|\alpha|^{4}+3|\alpha|^{2}+3 / 2\right)\right\} .
\end{aligned}
$$

Similarly, we get for $Y_{2 A}$ quadrature

$$
\begin{aligned}
{\left[\Delta Y_{2 A}(t)\right]^{2}-\left\langle N_{A}(t)+1 / 2\right\rangle=} & -40|g t||\alpha \beta| \sin \left(-\theta_{1}+\theta_{2}\right) \\
& \times\left\{\left(|\alpha|^{4}+3|\alpha|^{2}+3 / 2\right)\right\} .
\end{aligned}
$$

The right-hand sides of (36) and (37) are showing the existence of squeezing in amplitude-squared of the field in the fundamental mode in the fifth harmonic generation process whenever $\sin \left(-\theta_{1}+\theta_{2}\right)<0$ and $\sin \left(-\theta_{1}+\theta_{2}\right)>0$, respectively. The multiplication factor $\left(|\alpha|^{4}+3|\alpha|^{2}+3 / 2\right)$ is the nonlinear effect due to strong pump field interaction.

Now, we define the normalized parameter [30] for amplitude-squared squeezing as

$$
\begin{aligned}
Q_{y} & =\frac{\left[\Delta Y_{2 A}(t)\right]^{2}-\left\langle\left(N_{A}(t)+1 / 2\right)\right\rangle}{\left\langle\left(N_{A}(t)+1 / 2\right)\right\rangle} \\
& =\frac{-40|g t||\alpha \beta| \sin \left(-\theta_{1}+\theta_{2}\right)\left\{\left(|\alpha|^{4}+3|\alpha|^{2}+3 / 2\right)\right\}}{\left(|\alpha|^{2}+1 / 2\right)+10|g t|\left|\alpha^{5} \beta\right| \sin \left(\theta_{2}-5 \theta_{1}\right)} .
\end{aligned}
$$

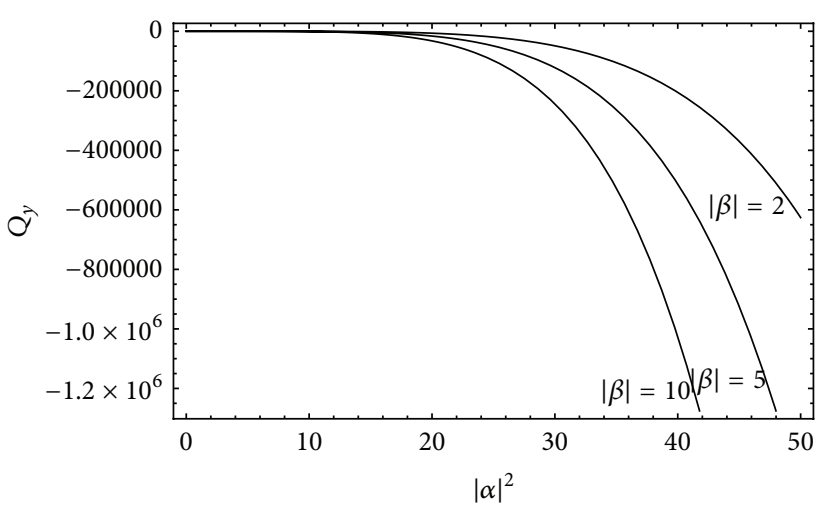

Figure 3: Dependence of degree of higher-order (amplitudesquared) squeezing $Q_{y}$ with $|\alpha|^{2}$ and $|\beta|$ in fifth harmonic generation (when $|g t|=10^{-4}$ and $\theta_{1}=0$ and $\theta_{2}=\pi / 2$ ).

Hence the degree of amplitude-squared (second order) squeezing in the fundamental mode depends directly upon the phase angle of the field amplitude up to the first-order Hamiltonian interaction in $g$ t. The variation of $Q_{y}$ with $|\alpha|^{2}$ is shown in Figure 3.

In Figure 3, the steady fall of the curve shows that the squeezing increases nonlinearly with $|\alpha|^{2}$, which depends directly upon the number of photons. This confirms that the reduction of quantum noise is associated with a large number of photons. We also find that when higher the value of $|\beta|$, then the squeezing increases and it lowers the depth of classicality of the field amplitude. It confirms that the degree of squeezing depends directly upon the photon number of the fundamental as well as of the harmonic field.

A comparison between Figures 2 and 3 shows greater noise reduction in higher order; that is, squeezing is maximum in amplitude-squared of the field (second order) than the first order having the same number of photons. Since higher-order squeezing (second order squeezing) is the higher powers of the field amplitude of the fundamental mode, which is directly associated with the large number of photons that it makes possible to achieve significantly larger signal noise reduction than the ordinary squeezing (normal or first-order squeezing).

Now, using (35), we obtain expectation values of mean photon number as

$$
\begin{aligned}
\left\langle N_{A}^{2}(t)\right\rangle= & |\alpha|^{4}+|\alpha|^{2}-5 i g t \\
& \times\left(2|\alpha|^{2} \alpha^{* 5} \beta-2|\alpha|^{2} \alpha^{5} \beta^{*}+5 \alpha^{* 5} \beta-5 \alpha^{5} \beta^{*}\right), \\
\left\langle N_{A}(t)\right\rangle^{2}= & |\alpha|^{4}-5 i g t\left(2|\alpha|^{2} \alpha^{* 5} \beta-2|\alpha|^{2} \alpha^{5} \beta^{*}\right) .
\end{aligned}
$$

Hence the fluctuation of time-dependent mean photon number is

$$
\begin{aligned}
{\left[\Delta N_{A}(t)\right]^{2} } & =\left\langle N_{A}^{2}(t)\right\rangle-\left\langle N_{A}(t)\right\rangle^{2} \\
& =|\alpha|^{2}-25 i g t\left(\alpha^{* 5} \beta-\alpha^{5} \beta^{*}\right),
\end{aligned}
$$


and the photon statistics of pump mode in fifth harmonic generation is found to be sub-Poissonian, as

$$
\left[\Delta N_{A}(t)\right]^{2}-\left\langle N_{A}(t)\right\rangle=40|g t|\left|\alpha^{5} \beta\right| \sin \left(\theta_{2}-5 \theta_{1}\right) .
$$

The right-hand side of (41) is always negative whenever $\sin \left(-5 \theta_{1}+\theta_{2}\right)<0$, showing the existence of sub-Poissonian light in fundamental mode up to first order Hamiltonian interaction in $g t$ under short-time approximation in the fifth harmonic generation process. It also indicates that the occurrence of sub-Poissonian light in the fundamental mode depends directly upon the phase angle of the field amplitude. These results are one of the distinguished examples of fifth harmonic generation process when light exhibits both squeezing and sub-Poissonian photon statistics at the same time up to first-order Hamiltonian interaction.

\section{Nonclassical Effects of Light in the Harmonic Mode}

It is of interest to examine squeezing in the harmonic mode $B$ as a function of time; we define the quadrature operators

$$
\begin{aligned}
& X_{1 B}(t)=\frac{1}{2}\left[B(t)+B^{\dagger}(t)\right], \\
& X_{2 B}(t)=\frac{1}{2 i}\left[B(t)-B^{\dagger}(t)\right] .
\end{aligned}
$$

Using (21) in (42), we find

$$
\begin{aligned}
& X_{1 B}(t)=\frac{1}{2}\left[\left(B+B^{\dagger}\right)-i g t\left(A^{5}-A^{\dagger^{5}}\right)\right], \\
& X_{2 B}(t)=\frac{1}{2 i}\left[\left(B-B^{\dagger}\right)-i g t\left(A^{5}+A^{\dagger^{5}}\right)\right] .
\end{aligned}
$$

Using (25) in (43), we obtain

$$
\begin{aligned}
& \left\langle\psi\left|X_{1 B}^{2}(t)\right| \psi\right\rangle \\
& =\frac{1}{4}\left[\beta^{2}+\beta^{* 2}+2|\beta|^{2}+1\right. \\
& \left.\quad-2 i g t\left(\alpha^{5} \beta+\alpha^{5} \beta^{*}-\alpha^{* 5} \beta-\alpha^{* 5} \beta^{*}\right)\right], \\
& \left\langle\psi\left|X_{1 B}(t)\right| \psi\right\rangle^{2} \\
& =\frac{1}{4}\left[\beta^{2}+\beta^{* 2}+2|\beta|^{2}\right. \\
& \left.\quad-2 i g t\left(\alpha^{5} \beta+\alpha^{5} \beta^{*}-\alpha^{* 5} \beta-\alpha^{* 5} \beta^{*}\right)\right] .
\end{aligned}
$$

Hence

$$
\left[\Delta X_{1 B}(t)\right]^{2}=\left\langle X_{1 B}^{2}(t)\right\rangle-\left\langle X_{1 B}(t)\right\rangle^{2}=\frac{1}{4} .
$$

Similarly, from (44), we have

$$
\left[\Delta X_{2 B}(t)\right]^{2}=\frac{1}{4} .
$$

From (46) and (47), we can see that the $B$-mode is initially in a coherent state; that is,

$$
\left[\Delta X_{1 B}\right]^{2}=\left[\Delta X_{2 B}\right]^{2}=\frac{1}{4}
$$

Hence there is no possibility to produce squeezed light in the harmonic mode up to first-order Hamiltonian interaction in $g t$.

It is now interesting to further study squeezing in the $B$ mode (harmonic) up to second-order Hamiltonian interaction in $g t$ of the fundamental mode.

From (17), we may obtain

$$
\ddot{B}=-g^{2}\left(G_{\{12\}}\right) B .
$$

Using (18) and the corresponding results in the amplitude harmonic mode is up to second order in " $g t$ ", we get

$$
\begin{gathered}
B(t)=B(0)-i g t A^{5}-\frac{1}{2}|g t|^{2}\left(G_{\{12\}}\right) B, \\
B^{\dagger}(t)=B^{\dagger}(0)+i g t A^{\dagger 5}-\frac{1}{2}|g t|^{2}\left(G_{\{12\}}\right) B^{\dagger} .
\end{gathered}
$$

Using (50) and (8) in (42), we get

$$
\begin{aligned}
& X_{1 B}(t)=X_{1 B}+g t G_{2 A}-\frac{1}{2}|g t|^{2}\left(G_{\{12\}}\right) X_{1 B}, \\
& X_{2 B}(t)=X_{2 B}-g t G_{1 A}-\frac{1}{2}|g t|^{2}\left(G_{\{12\}}\right) X_{2 B} .
\end{aligned}
$$

For uncorrelated modes at $t=0$, we get the field variance in harmonic mode as

$$
\begin{aligned}
{\left[\Delta X_{1 B}(t)\right]^{2}=} & \left(\Delta X_{1 B}\right)^{2}+|g t|^{2} \\
& \times\left[\left(\Delta G_{2 A}\right)^{2}-\left\langle\left(G_{\{12\}}\right)\right\rangle\left(\Delta X_{1 B}\right)^{2}\right] \\
{\left[\Delta X_{2 B}(t)\right]^{2}=} & \left(\Delta X_{2 B}\right)^{2}+|g t|^{2} \\
& \times\left[\left(\Delta G_{1 A}\right)^{2}-\left\langle\left(G_{\{12\}}\right)\right\rangle\left(\Delta X_{2 B}\right)^{2}\right] .
\end{aligned}
$$

Using (48), then we have

$$
\begin{aligned}
& {\left[\Delta X_{1 B}(t)\right]^{2}-\frac{1}{4}=|g|^{2} t^{2}\left[\left(\Delta G_{2 A}\right)^{2}-\frac{1}{4}\left\langle\left(G_{\{12\}}\right)\right\rangle\right]} \\
& {\left[\Delta X_{2 B}(t)\right]^{2}-\frac{1}{4}=|g|^{2} t^{2}\left[\left(\Delta G_{1 A}\right)^{2}-\frac{1}{4}\left\langle\left(G_{\{12\}}\right)\right\rangle\right] .}
\end{aligned}
$$

Equations (53) and (54) show that $X_{1 B}$ in the harmonic mode is squeezed if $G_{2 A}$ is squeezed and $X_{2 B}$ is squeezed if $G_{1 A}$ is squeezed. In other words, the harmonic mode is squeezed in the $X_{1 B}$ direction if the fundamental mode is fifth-order squeezing in the $G_{2 A}$ direction and the harmonic mode is squeezed in the $X_{2 B}$ direction if the fundamental mode is fifth-order squeezing in the $G_{1 A}$ direction. That is, if a fundamental mode with fifth-order squeezing propagates through a nonlinear medium, a squeezed harmonic mode is generated. 
Now, comparing the results of (54) with (A.11) [see Appendix for details of the calculation of (A.11)], then we have (we consider here only the $G_{1 A}(t)$ quadrature)

$$
\begin{aligned}
{\left[\Delta X_{2 B}(t)\right]^{2}-\frac{1}{4} } \\
=-\left(\frac{5}{4}\right)|g t|^{4} \\
\quad \times\left\{\left(10|\alpha|^{18}+2800|\alpha|^{16}+26400|\alpha|^{14}\right.\right. \\
\left.\quad+97680|\alpha|^{12}+116880|\alpha|^{10}\right) \cos (10 \theta) \\
+10|\alpha|^{18}+430|\alpha|^{16}+8690|\alpha|^{14}+94343|\alpha|^{12} \\
\quad+514138|\alpha|^{10}+1389600|\alpha|^{8}+1756800|\alpha|^{6} \\
\left.\quad+936000|\alpha|^{4}+149760|\alpha|^{2}+5760\right\} .
\end{aligned}
$$

The right hand side of (55) shows that the squeezing in the harmonic mode is $|g t|^{2}$ times more than the fifth-order amplitude squeezing in the fundamental mode in the fifth harmonic generation process. This establishes the fact that the occurrence of squeezing in the harmonic mode is only due to the presence of the fundamental mode. An analysis of (55) shows that if $g^{2} t^{2}<1$, squeezing is greater in harmonic mode compared to fundamental mode. Further, a comparison between the results of (30), (38), (41) and (55) with our earlier work [42] it is observed that the squeezing and sub-Poissonian effects of light are more pronounced to reduce the quantum noise in the fifth harmonic generation than the corresponding squeezing and sub-Poissonian effects of light in the fourth harmonic generation.

To study optimum squeezing in harmonic mode, we denote the right-hand side of (55) by $S_{X}$, and taking $|g t|^{2}=$ $10^{-4}$ and $\theta=0$. The variation of $S_{X}$ with $|\alpha|^{2}$ is shown in Figure 4.

Figure 4 shows that the squeezing increases nonlinearly with $|\alpha|^{2}$, which is directly dependent upon the number of photons. This again confirms that the squeezed states are associated with a large number of photons. Moreover, the curve also establishes the relationship between the harmonic mode and the fundamental mode.

\section{Conclusions}

The important results of this paper can be listed as follows.

(i) The existence of amplitude squeezing in $X_{1 A}$ or $X_{2 A}$ quadrature up to the first order Hamiltonian interaction in $g t$ in the fundamental mode will occur whenever $\sin \left(\theta_{2}-3 \theta_{1}\right)<0$ or $\sin \left(\theta_{2}-3 \theta_{1}\right)>$ 0 respectively. Similarly the existence of amplitudesquared i.e. second-order squeezing in $Y_{1 \mathrm{~A}}$ or $Y_{2 \mathrm{~A}}$ quadrature of the fundamental mode will occur whenever $\sin (-\theta+\theta)<0$ or $\sin -\left(\theta_{1}+\theta_{2}\right)>$ 0 under short-time approximation in the fifth harmonic generation process. The multiplication factor

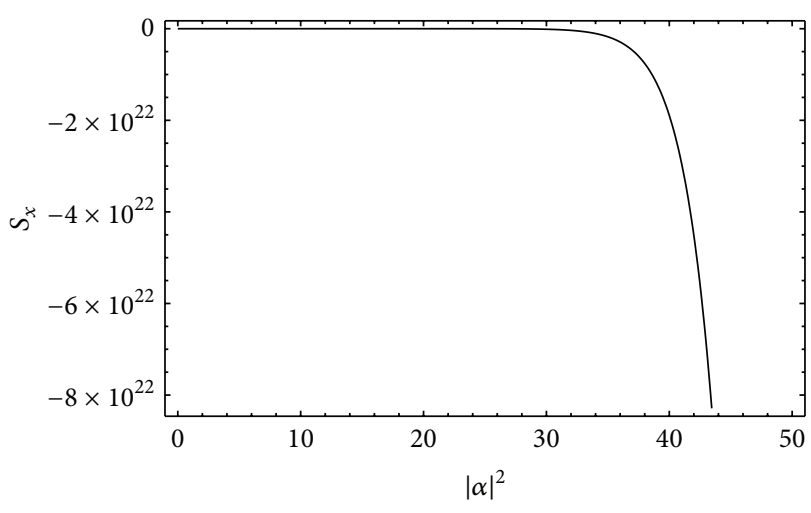

FIGURE 4: Dependence of harmonic squeezing $S_{x}$ with $|\alpha|^{2}$ in fifth harmonic generation (when $|g t|^{2}=10^{-4}$ and $\theta=0$ ).

$\left(|\alpha|^{4}+3|\alpha|^{2}+3 / 2\right)$ in the second-order squeezing is the nonlinear effect due to strong pump field interaction.

(ii) In the fifth harmonic generation process up to the first-order Hamiltonian interaction in $g t$, the subPoissonian photon statistics exists in the fundamental mode when $\sin \left(-5 \theta_{1}+\theta_{2}\right)<0$.

(iii) We have found that the degree of squeezing and subPoissonian photon statistics in the fundamental mode are directly dependent upon the selective phase values of the field amplitude up to the first-order interaction in $g t$ in the fifth harmonic generation process.

(iv) It is observed that when higher the value of $|\beta|$, then squeezing increases and it lowers the depth of classicality of the field amplitude. The degree of squeezing is found to be dependent on the photon number of fundamental field as well as on the harmonic field.

(v) There is no possibility to produce squeezed laser light in the harmonic mode up to first-order Hamiltonian interaction in $g t$.

(vi) The Hamiltonian interaction up to second order in gt revealed that the harmonic mode is squeezed in the $X_{1 B}$ direction if the fundamental mode is fifth-order squeezing in the $G_{2 A}$ direction and the harmonic-mode is squeezed in the $X_{2 B}$ direction if the fundamental mode is fifth-order squeezing in the $G_{1 A}$ direction. That is, the squeezing in the harmonic mode depends on the fifth-order squeezing of the fundamental mode. This gives a method of converting higher-order squeezing into normal squeezing in harmonic mode.

(vii) We have observed the fact that the occurrence of amplitude squeezing in the harmonic mode is only due to the presence of the fundamental mode.

(viii) In an analysis of (55), it is observed that squeezing in the harmonic mode is $|g t|^{2}$ times more than the fifth-order amplitude squeezing in the fundamental mode in the fifth harmonic generation process. This fact established the relationship between fundamental and harmonic mode. It is found that if $g^{2} t^{2}<1$ 
squeezing is greater in harmonic mode compared to fundamental mode.

(ix) Moreover, we have found that the squeezing increases nonlinearly with $|\alpha|^{2}$, which is directly dependent upon the number of photons. This has confirmed that the squeezed states (reduction of quantum noise) are associated with a large number of photons.

(x) One of the higher-order squeezing in fifth harmonic generation process, that is, fifth-order squeezing, is found to be more squeezed than the fourth-order squeezing [43]. Hence, the higher-order squeezing makes it possible to achieve significantly larger noise reduction than lower-order or ordinary squeezing.

(xi) The maximum reachable degree of squeezing is directly dependent upon the short interaction time as well as the number of photons. However, the maximum attainable squeezing will be limited by short interaction time.

(xii) It is observed that the squeezing and sub-Poissonian effects of light are more pronounced to reduce the quantum noise in the fifth harmonic generation than the corresponding squeezing and sub-Poissonian effects of light in the fourth harmonic generation [42].

As a result, the family of nonclassical effects like squeezing and sub-Poissonian photon statistics in relation to selective phase values of the field amplitude up to the first-order Hamiltonian interaction in $g t$ are useful to obtain the desired degree of amplitude squeezing through different higherorder nonlinear optical processes. Hence, these results may pave the way for obtaining greater noise reduction in any optical systems and can be useful in high quality quantum telecommunication.

\section{Appendix}

Expanding $A(t)$ around the initial time $t=0$ in Taylor's expansion (18) and confining ourselves to terms up to second order in " $g t$ ", we obtain

$$
\begin{aligned}
A(t)= & A-5 i g t A^{\dagger 4} B+\frac{5}{2} g^{2} t^{2} \\
\times & {\left[5\left(4 A^{\dagger 3} A^{4}+24 A^{\dagger 2} A^{3}+48 A^{\dagger} A^{2}+24 A\right) B^{\dagger} B\right.} \\
& \left.\quad-A^{\dagger 4} A^{5}\right] .
\end{aligned}
$$

Now, we assume an initial quantum state as a product of coherent states $\mid \alpha>$ for the fundamental mode $A$ and vacuum state $\mid 0>$ for the harmonic mode $B$; that is,

$$
|\psi>=| \alpha>_{A} \mid 0>_{B}
$$

Using (A.2), then (A.1) reduces to

$$
\langle\psi|A(t)| \psi\rangle=\left\langle\psi\left|A-\frac{5}{2} g^{2} t^{2}\left[A^{\dagger 4} A^{5}\right]\right| \psi\right\rangle .
$$

And in reversal order, consider

$$
\left\langle\psi\left|A^{\dagger}(t)\right| \psi\right\rangle=\left\langle\psi\left|A^{\dagger}-\frac{5}{2} g^{2} t^{2}\left[A^{\dagger 5} A^{4}\right]\right| \psi\right\rangle .
$$

Using (A.3) and (A.4) in (8) for the fundamental mode $A$, the fifth-order squeezing of $G_{1 A}$ quadrature may be written as

$$
\begin{aligned}
& \left\langle\psi\left|G_{1 A}(t)\right| \psi\right\rangle \\
& =\frac{1}{2}\left[\alpha^{5}+\alpha^{* 5}-\frac{5}{2}|g|^{2} t^{2}\right. \\
& \quad \times\left(5 \alpha^{* 4} \alpha^{9}+40 \alpha^{* 3} \alpha^{8}+120 \alpha^{* 2} \alpha^{7}+84 \alpha^{*} \alpha^{6}\right. \\
& +24 \alpha^{5}+5 \alpha^{* 9} \alpha^{4}+40 \alpha^{* 8} \alpha^{3}+120 \alpha^{* 7} \alpha^{2} \\
& \left.\left.+84 \alpha^{* 6} \alpha+24 \alpha^{* 5}\right)\right] .
\end{aligned}
$$

Utilizing (A.5), we obtain the expectation value of the square of the field amplitude operator as

$$
\begin{aligned}
&\left\langle\psi\left|G_{1 A}^{2}(t)\right| \psi\right\rangle \\
&=\frac{1}{4}\left[\alpha^{10}+\alpha^{* 10}+2 \alpha^{* 5} \alpha^{5}+25 \alpha^{* 4} \alpha^{4}+200 \alpha^{* 3} \alpha^{3}\right. \\
&+600 \alpha^{* 2} \alpha^{2}+600 \alpha^{*} \alpha+120-5|g|^{2} t^{2} \\
& \times\left(10 \alpha^{* 4} \alpha^{14}+180 \alpha^{* 3} \alpha^{13}+1440 \alpha^{* 2} \alpha^{12}\right. \\
&+4968 \alpha^{*} \alpha^{11}+5868 \alpha^{10}+10 \alpha^{* 14} \alpha^{4} \\
&+180 \alpha^{* 13} \alpha^{3}+1440 \alpha^{* 12} \alpha^{2}+4968 \alpha^{* 11} \alpha \\
&+5868 \alpha^{* 10}+20 \alpha^{* 9} \alpha^{9}+610 \alpha^{* 8} \alpha^{8} \\
&+10880 \alpha^{* 7} \alpha^{7}+103936 \alpha^{* 6} \alpha^{6}+525936 \alpha^{* 5} \alpha^{5} \\
&+1389600 \alpha^{* 4} \alpha^{4}+1756800 \alpha^{* 3} \alpha^{3} \\
&\left.\left.+936000 \alpha^{* 2} \alpha^{2}+149760 \alpha^{*} \alpha+5760\right)\right]
\end{aligned}
$$

And the square of the expectation value of the field operator is as follows:

$$
\begin{aligned}
& \left\langle\psi\left|G_{1 A}(t)\right| \psi\right\rangle^{2} \\
& =\frac{1}{4}\left[\alpha^{10}+\alpha^{* 10}+2 \alpha^{* 5} \alpha^{5}-5|g|^{2} t^{2}\right. \\
& \times\left(5 \alpha^{* 4} \alpha^{14}+40 \alpha^{* 3} \alpha^{13}+120 \alpha^{* 2} \alpha^{12}+84 \alpha^{*} \alpha^{11}\right. \\
& +24 \alpha^{10}+5 \alpha^{* 14} \alpha^{4}+40 \alpha^{* 13} \alpha^{3}+120 \alpha^{* 12} \alpha^{2} \\
& +84 \alpha^{* 11} \alpha+24 \alpha^{* 10}+10 \alpha^{* 9} \alpha^{9}+80 \alpha^{* 8} \alpha^{8} \\
& \left.\left.+240 \alpha^{* 7} \alpha^{7}+168 \alpha^{* 6} \alpha^{6}+486 \alpha^{* 5} \alpha^{5}\right)\right] .
\end{aligned}
$$


Hence the field variance is

$$
\begin{aligned}
& {\left[\Delta G_{1 A}(t)\right]^{2}} \\
& =\left\langle G_{1 A}^{2}(t)\right\rangle-\left\langle G_{1 A}(t)\right\rangle^{2} \\
& =\frac{1}{4}\left[25 \alpha^{* 4} \alpha^{4}+200 \alpha^{* 3} \alpha^{3}+600 \alpha^{* 2} \alpha^{2}+600 \alpha^{*} \alpha+120\right. \\
& -5|g|^{2} t^{2}\left(5 \alpha^{* 4} \alpha^{14}+140 \alpha^{* 3} \alpha^{13}+1320 \alpha^{* 2} \alpha^{12}\right. \\
& +4884 \alpha^{*} \alpha^{11}+5844 \alpha^{10}+5 \alpha^{* 14} \alpha^{4} \\
& +140 \alpha^{* 13} \alpha^{3}+1320 \alpha^{* 12} \alpha^{2}+4884 \alpha^{* 11} \alpha \\
& +5844 \alpha^{* 10}+10 \alpha^{* 9} \alpha^{9}+530 \alpha^{* 8} \alpha^{8} \\
& +10640 \alpha^{* 7} \alpha^{7}+103768 \alpha^{* 6} \alpha^{6} \\
& +525888 \alpha^{* 5} \alpha^{5}+1389600 \alpha^{* 4} \alpha^{4} \\
& +1756800 \alpha^{* 3} \alpha^{3}+936000 \alpha^{* 2} \alpha^{2} \\
& \left.\left.+149760 \alpha^{*} \alpha+5760\right)\right] .
\end{aligned}
$$

Now, using (A.3) and (A.4), we obtain

$$
\begin{aligned}
\left\langle\psi\left|N_{A}(t)\right| \psi\right\rangle & =\left\langle\psi\left|A^{\dagger}(t) A(t)\right| \psi\right\rangle \\
& =|\alpha|^{2}-5|g|^{2} t^{2}\left(\alpha^{* 5} \alpha^{5}\right) .
\end{aligned}
$$

Similarly, we can derive the higher expectation value of the mean photon number $\left\langle\psi\left|N_{A}^{2}(t)\right| \psi\right\rangle,\left\langle\psi\left|N_{A}^{3}(t)\right| \psi\right\rangle$, $\left\langle\psi\left|N_{A}^{4}(t)\right| \psi\right\rangle$.

Utilizing all expectation values of the mean photon number, we get

$$
\begin{aligned}
\frac{1}{4}\left\langle\left(G_{\{12\}}\right)\right\rangle=\frac{1}{4}[ & 25 \alpha^{* 4} \alpha^{4}+200 \alpha^{* 3} \alpha^{3}+600 \alpha^{* 2} \alpha^{2} \\
& +600 \alpha^{*} \alpha+120-5|g|^{2} t^{2} \\
& \times\left(100 \alpha^{* 8} \alpha^{8}+1950 \alpha^{* 7} \alpha^{7}\right. \\
& \left.\left.+9425 \alpha^{* 6} \alpha^{6}+11750 \alpha^{* 5} \alpha^{5}\right)\right]
\end{aligned}
$$

Using (A.8) and (A.10), we obtain

$$
\begin{aligned}
{\left[\Delta G_{1 A}(t)\right]^{2}-\frac{1}{4}\left\langle\left(G_{\{12\}}\right)\right\rangle } & \\
=-\left(\frac{5}{4}\right)|g t|^{2}\{ & \left(10|\alpha|^{18}+2800|\alpha|^{16}+26400|\alpha|^{14}\right. \\
& \left.+97680|\alpha|^{12}+116880|\alpha|^{10}\right) \cos (10 \theta) \\
& +10|\alpha|^{18}+430|\alpha|^{16}+8690|\alpha|^{14}
\end{aligned}
$$

$$
\begin{aligned}
& +94343|\alpha|^{12}+514138|\alpha|^{10} \\
& +1389600|\alpha|^{8}+1756800|\alpha|^{6} \\
& \left.+936000|\alpha|^{4}+149760|\alpha|^{2}+5760\right\} .
\end{aligned}
$$

The right-hand side of (A.11) is always negative, showing the existence of squeezing in fifth power of the field amplitude of the fundamental mode in fifth harmonic generation process. The result is found to be more squeezed than the fourth-order squeezing [43].

\section{Conflict of Interests}

The authors declare that there is no conflict of interests regarding the publication of this paper.

\section{Acknowledgment}

R. S. More College, Sindri College, and P. K. R. M. College are Constituent Units of Vinoba Bhave University, Hazaribag.

\section{References}

[1] D. F. Walls, “Squeezed states of light," Nature, vol. 306, no. 5939, pp. 141-146, 1983.

[2] R. Loudon and P. L. Knight, "Squeezed light," Journal of Modern Optics, vol. 34, no. 6-7, pp. 709-759, 1987.

[3] M. C. Teich and B. E. A. Saleh, "Squeezed state of light," Quantum Optics B, vol. 1, no. 2, pp. 153-191, 1989.

[4] J. Perina, Quantum Statistics of Linear and Nonlinear Optical Phenomena, chapters 9 and 10, Kluwer, Dordrecht, The Netherlands, 1991.

[5] L. Mandel, "Nonclassical states of the electromagnetic field," Physica Scripta T, vol. 12, pp. 34-42, 1986.

[6] V. V. Dodonov, "Nonclassical states in quantum optics: a squeezed review of the first 75 years," Journal of Optics B, vol. 4, no. 1, pp. R1-R33, 2002.

[7] B. E. A. Saleh and M. C. Teich, "Can the channel capacity of a light-wave communication system be increased by the use of photon-number-squeezed light?" Physical Review Letters, vol. 58 , no. 25 , pp. 2656-2659, 1987.

[8] K. Wódkiewicz, "On the quantum mechanics of squeezed states," Journal of Modern Optics, vol. 34, pp. 941-948, 1987.

[9] H. J. Kimble and D. F. Walls, "Squeezed states of the electromagnetic field: introduction to feature issue," Journal of the Optical Society of America B, vol. 4, pp. 1450-1741, 1987.

[10] H. P. Yuen and J. H. Shapiro, "Optical communication with twophoton coherent states-part I: quantum-state propagation and quantum-noise reduction," IEEE Transactions on Information Theory, vol. 24, no. 6, pp. 657-668, 1978.

[11] C. H. Bennett, G. Brassard, and N. D. Mermin, "Quantum cryptography without Bell's theorem," Physical Review Letters, vol. 68 , no. 5, pp. 557-559, 1992.

[12] J. Kempe, "Multiparticle entanglement and its applications to cryptography," Physical Review A, vol. 60, no. 2, pp. 910-916, 1999.

[13] L. Mandel, "Squeezing and photon antibunching in harmonic generation," Optics Communications, vol. 42, no. 6, pp. 437-439, 1982. 
[14] S. Kielich, R. Tanas, and R. Zawodny, "Squeezing in the thirdharmonic field generated by self-squeezed light," Journal of the Optical Society of America B, vol. 4, pp. 1627-1632, 1987.

[15] J. Peřina, V. Peřinová, C. Sibilia, and M. Bertolotti, "Quantum statistics of four-wave mixing," Optics Communications, vol. 49, no. 4, pp. 285-289, 1984.

[16] M. S. K. Razmi and J. H. Eberly, "Degenerate four-wave mixing and squeezing in pumped three-level atomic systems," Optics Communications, vol. 76, no. 3-4, pp. 265-267, 1990.

[17] D. K. Giri and P. S. Gupta, "The squeezing of radiation in fourwave mixing processes," Journal of Optics B, vol. 6, no. 1, pp. 9196, 2004.

[18] D. K. Giri and P. S. Gupta, "Short-time squeezing effects in spontaneous and stimulated six-wave mixing process," Optics Communications, vol. 221, no. 1-3, pp. 135-143, 2003.

[19] J. Perina and J. Krepelka, "Stimulated Raman scattering of squeezed light with pump depletion," Journal of Modern Optics, vol. 38, no. 11, pp. 2137-2151, 1991.

[20] A. Kumar and P. S. Gupta, "Short-time squeezing in spontaneous Raman and stimulated Raman scattering," Quantum and Semiclassical Optics B, vol. 7, no. 5, pp. 835-841, 1995.

[21] A. Kumar and P. S. Gupta, "Higher-order amplitude squeezing in hyper-Raman scattering under short-time approximation," Journal of Optics B, vol. 8, no. 5, pp. 1053-1060, 1996.

[22] D. K. Giri and P. S. Gupta, "Higher-order squeezing of the electromagnetic field in spontaneous and stimulated Raman processes," Journal of Modern Optics, vol. 52, no. 12, pp. 17691781, 2005.

[23] C. K. Hong and L. Mandel, "Higher-order squeezing of a quantum field," Physical Review Letters, vol. 54, no. 4, pp. 323$325,1985$.

[24] C. K. Hong and L. Mandel, "Generation of higher-order squeezing of quantum electromagnetic fields," Physical Review A, vol. 32, no. 2, pp. 974-982, 1985.

[25] M. Hillery, "Squeezing of the square of the field amplitude in second harmonic generation," Optics Communications, vol. 62, no. 2, pp. 135-138, 1987.

[26] M. Hillery, "Amplitude-squared squeezing of the electromagnetic field," Physical Review A, vol. 36, no. 8, pp. 3796-3802, 1987.

[27] M. Hillery, "Phase-space representation of amplitude-squared squeezing," Physical Review A, vol. 45, no. 7, pp. 4944-4950, 1992.

[28] Y.-B. Zhan, "Amplitude-cubed squeezing in harmonic generations," Physics Letters A, vol. 160, no. 6, pp. 498-502, 1991.

[29] J. Peěrina, V. Peěrinová, and J. Kod’ousek, "On the relations of antibunching, sub-poissonian statistics and squeezing," Optics Communications, vol. 49, no. 3, pp. 210-214, 1984.

[30] Y. Kim and T. H. Yoon, "Higher order sub-Poissonian photon statistics of light," Optics Communications, vol. 212, no. 1-3, pp. 107-114, 2002.

[31] H. Prakash and D. K. Mishra, "Higher order sub-Poissonian photon statistics and their use in detection of Hong and Mandel squeezing and amplitude-squared squeezing," Journal of Physics B, vol. 39, no. 9, pp. 2291-2297, 2006.

[32] D. K. Mishra, "Study of higher order non-classical properties of squeezed Kerr state," Optics Communications, vol. 283, no. 17, pp. 3284-3290, 2010.

[33] S. Gill, S. Rani, and N. Singh, "Higher order amplitude squeezing in fourth and fifth harmonic generation," Indian Journal of Physics, vol. 86, pp. 371-375, 2012.
[34] N. B. An and V. Tinh, "General multimode sum-squeezing," Physics Letters A, vol. 261, no. 1-2, pp. 34-39, 1999.

[35] N. B. An and V. Tinh, "General multimode differencesqueezing," Physics Letters A, vol. 270, no. 1-2, pp. 27-40, 2000.

[36] L. Mandel, "Sub-Poissonian photon statistics in resonance fluorescence," Optics Letters, vol. 4, pp. 205-207, 1979.

[37] L.-B. Chang, S. C. Wang, and A. H. Kung, "Numerical analysis of fifth-harmonic conversion of low-power pulsed Nd:YAG laser with resonance of second harmonic," Japanese Journal of Applied Physics A, vol. 42, no. 7A, pp. 4318-4324, 2003.

[38] J. Ni, J. Yao, B. Zeng et al., "Comparative investigation of thirdand fifth-harmonic generation in atomic and molecular gases driven by midinfrared ultrafast laser pulses," Physical Review A, vol. 84, no. 6, Article ID 063846, 4 pages, 2011.

[39] R. Tanaś, "Squeezing from an anharmonic oscillator model: $\left(a^{+}\right)^{2} a^{2}$ versus $\left(a^{+} a\right)^{2}$ interaction Hamiltonians," Physics Letters A, vol. 141, no. 5-6, pp. 217-220, 1989.

[40] R. Tanas, A. Miranowicz, and S. Kielich, "Squeezing and its graphical representations in the anharmonic oscillator model," Physical Review A, vol. 43, no. 7, pp. 4014-4021, 1991.

[41] I. V. Kityk, A. Fahmi, B. Sahraoui, G. Rivoire, and I. Feeks, "Nitrobenzene as a material for the fast-respond degenerate four-wave mixing," Optical Materials, vol. 16, no. 4, pp. 417-429, 2001.

[42] R. Pratap, D. K. Giri, and A. Prasad, "Effects of Squeezing and sub-poissonian of light in fourth harmonic generation upto first-order Hamiltonian interaction," Optik, vol. 125, no. 3, pp. 1065-1070, 2014.

[43] S. Rani, J. Lal, and N. Singh, "Squeezing and sub-Poissonian effects up-to fourth order in fifth harmonic generation," Optics Communications, vol. 281, no. 2, pp. 341-346, 2008. 

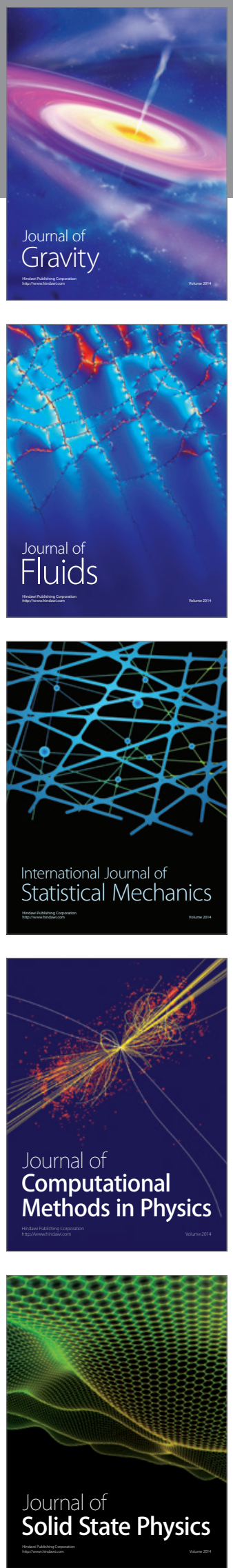

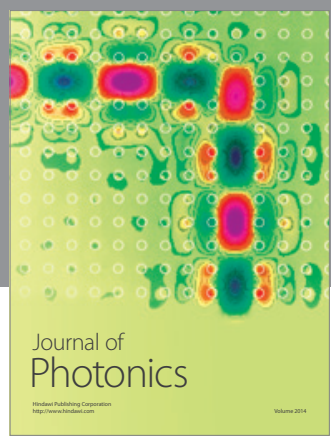

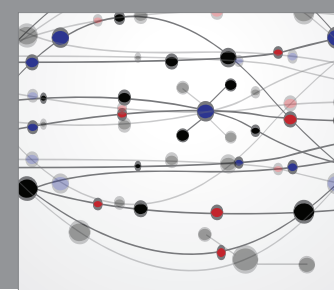

The Scientific World Journal

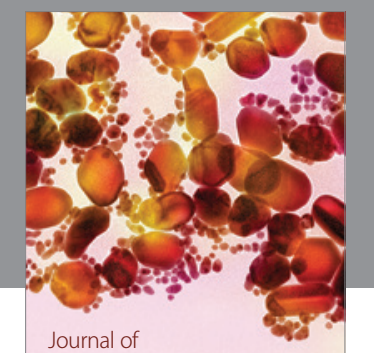

Soft Matter
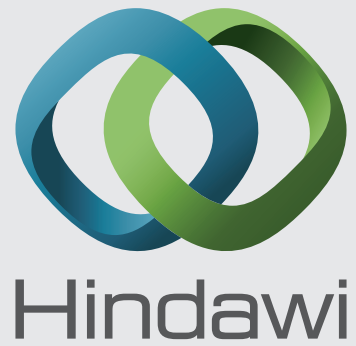

Submit your manuscripts at

http://www.hindawi.com
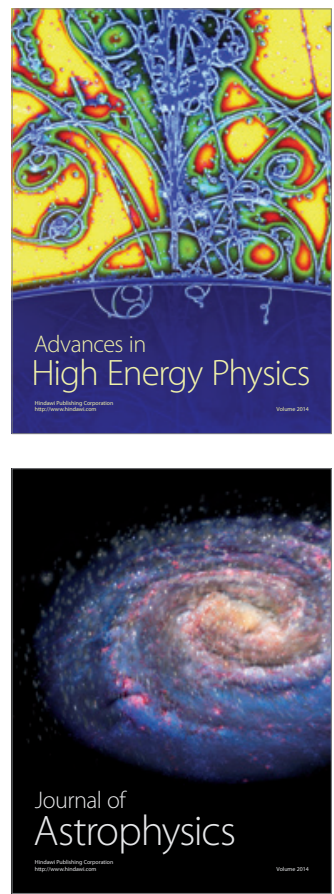
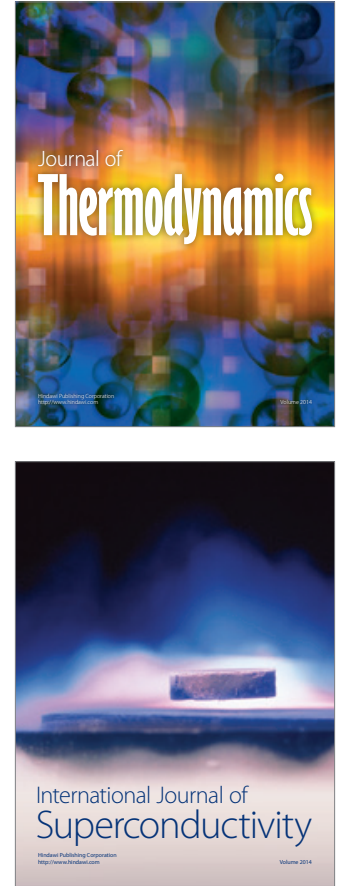
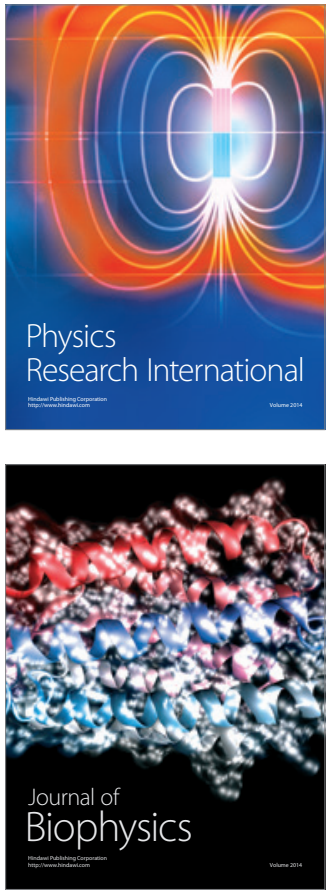
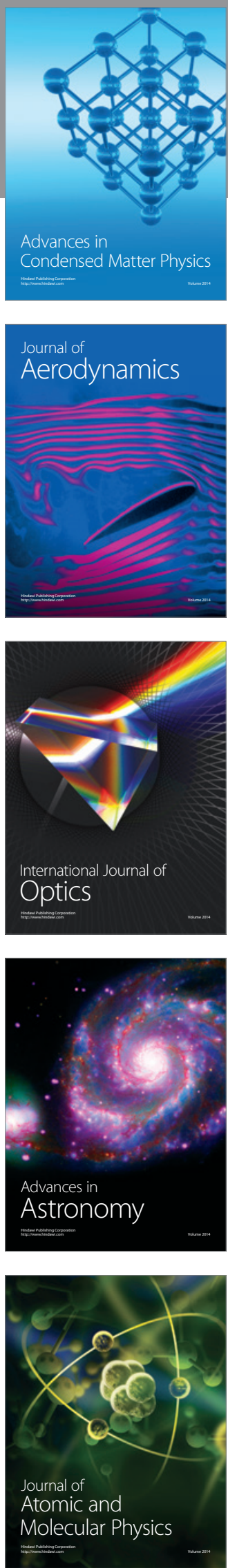